\title{
Interplay of CR-driven galactic wind, magnetic field, and galactic dynamo in spiral galaxies
}

\author{
Marita Krause \\ Max-Planck-Institut für Radioastronomie, Auf dem Hügel 69, 53121 Bonn, Germany \\ email: mkrause@mpifr-bonn.mpg.de
}

\begin{abstract}
From our radio observations of the magnetic field strength and large-scale pattern of spiral galaxies of different Hubble types and star formation rates (SFR) we conclude that though a high SFR in the disk increases the total magnetic field strength in the disk and the halo - the SFR does not change the global field configuration nor influence the global scale heights of the radio emission. The similar scale heights indicate that the total magnetic field regulates the galactic wind velocities. The galactic wind itself may be essential for an effective dynamo action.
\end{abstract}

Keywords. Galaxies: spiral - magnetic fields - halos - radio continuum: galaxies

\section{Magnetic field strength and star formation}

Observations of a sample of three late-type galaxies with low surface-brightness and the radio-weak edge-on galaxy NGC 5907 (all with a low SFR) revealed that they all have an unusually high thermal fraction and weak total and regular magnetic fields (Chyży et al. 2007, Dumke et al. 2000). However, these objects still follow the total radio-FIR correlation, extending it to the lowest values measured so far. Hence, these galaxies have a lower fraction of synchrotron emission than galaxies with higher SFR. It is known that the thermal intensity is proportional to the SFR. Our findings fit to the equipartition model for the radio-FIR correlation (Niklas \& Beck 1997), according to which the nonthermal emission increases $\propto S F R^{1.3 \pm 0.2}$ and the total magnetic field strength $\mathrm{B}_{\mathrm{t}}$ increases $\propto S F R^{0.34 \pm 0.14}$.

No similar simple relation exists for the regular magnetic field strength. We integrated the polarization properties in 41 nearby spiral galaxies and found that (independently of inclination effects) the degree of polarization is lower $(<4 \%)$ for more luminous galaxies, in particular those for $L_{4.8}>2 \times 10^{21} \mathrm{WHz}^{-1}$ (Stil et al. 2008). The radio-brightest galaxies are those with the highest SFR. Though a dynamo action needs star formation and supernova remnants as the driving force for velocities in vertical direction, we conclude from our observations that stronger star formation seems to reduce the magnetic field regularity. On kpc-scales, Chyży (2008) analyzed the correlation between magnetic field regularity and SFR locally within one galaxy, NGC 4254. While he found that the total and random field strength increase locally with SFR, the regular field strength is locally uncorrelated with SFR.

\section{Vertical scale heights and CR-driven galactic wind}

We determined the exponential scale heights of the total power emission at $\lambda 6 \mathrm{~cm}$ for four edge-on galaxies (NGC 253, NGC 891, NGC 3628, NGC 4565) for which we have 
combined interferometer and single-dish data (VLA and the 100-m Effelsberg). In spite of their different intensities and extents of the radio emission, the vertical scale heights of the thin disk and the thick disk/halo are similar in this sample (300 pc and $1.8 \mathrm{kpc})$ (Dumke \& Krause 1998, Heesen et al. 2009). We stress that our sample includes the brightest halo observed so far, NGC 253, with a very high SFR, as well as one of the weakest halos, NGC 4565, with a small SFR.

For NGC 253 Heesen et al. (this volume) argued that the synchrotron lifetime (which is $\propto \mathrm{B}_{\mathrm{t}}^{-2}$ ) mainly determines the vertical scale height of the synchrotron emission and estimated the cosmic ray bulk velocity to $300 \pm 30 \mathrm{~km} / \mathrm{s}$. As this is similar to the escape velocity, it shows the presence of a galactic wind in this galaxy. The fact that we observe similar averaged scaleheights at $\lambda 6 \mathrm{~cm}$ for the four galaxies mentioned above imply that the galactic wind velocity is proportional to $\mathrm{B}_{\mathrm{t}}^{2}$, and hence proportional to $\mathrm{SFR}^{0.7 \pm 0.3}$.

\section{Magnetic field structure, dynamo action, and galactic wind}

In a larger sample of 11 edge-on galaxies we found in all of them (except the inner part of NGC 4631, see Krause 2009) mainly a disk-parallel magnetic field along the galactic midplane together with an X-shaped poloidal field in the halo. Our sample includes spiral galaxies of different Hubble types and SFR, ranging from $0.5 \mathrm{M}_{\odot} \mathrm{yr}^{-1} \leqslant$ $\mathrm{SFR} \leqslant 27 \mathrm{M}_{\odot} \mathrm{yr}^{-1}$. The disk-parallel magnetic field is the expected edge-on projection of the spiral magnetic field within the disk as observed in face-on galaxies. It is generally thought to be generated by a mean-field $\alpha \Omega$-dynamo for which the most easily excited field pattern is the axismmetric spiral (ASS) field (e.g. Beck et al. 1996). The poloidal part of the ASS dynamo field alone, however, cannot explain the observed X-shaped structures in edge-on galaxies as the field strength there seems to be comparable to that of the large-scale disk field. Model calculations of the mean-field $\alpha \Omega$-dynamo for a disk surrounded by a spherical halo including a galactic wind (Brandenburg et al. 1993) simulated similar field configurations as the observed ones. New MHD simulations are in progress (see e.g. Gressel et al. this volume, Hanasz et al. this volume) which include a galactic wind implicitely. A galactic wind can also solve the helicity problem of dynamo action (e.g. Sur et al. 2007). Hence, a galactic wind may be essential for an effective dynamo action, and to explain the observed similar vertical scale heights and X-shaped magnetic field structure in edge-on galaxies.

\section{References}

Beck, R., Brandenburg, A., Moss, D., Shukurov, A., \& Sokoloff, D. D. 1996, ARAA 34, 155

Brandenburg, A., Donner, K. J., Moss, D., Shukurov, A., Sokoloff, \& D. D., Tuominen, I. 1993, $A \mathscr{E} A 271,36$

Chyży, K. T., Bomans, D. J., Krause, M., Beck, R., Soida, M., \& Urbanik, M. 2007, A\&\&A 462, 933

Chyży, K. T. 2008, A\&̊A 482, 755

Dumke, M. \& Krause, M. 1998, in: D. Breitschwerdt, M. Freyberg \& J. Trümper (eds.), The Local Bubble and Beyond, Lecture Notes in Physics (Heidelberg: Springer), vol. 166, p. 555

Dumke, M., Krause, M., \& Wielebinski, R. 2000, A\&A 355, 512

Heesen, V., Beck, R., Krause, M., \& Dettmar, R.-J. 2009, A\&AA, in press, arXiv:astro$\mathrm{ph} / 0812.0346$

Krause, M. 2009, Rev. Mexicana AyA, in press, arXiv:astro-ph/0806.2060

Niklas, S. \& Beck, R. 1997, A\&\&A 320, 54

Stil, J. M., Krause, M., Beck, R., \& Taylor, A. R. 2008, A\&SA, in press, arXiv:astro-ph/0810.2303

Sur, S., Shukurov, A., \& Subramanian, K. 2007, MNRAS 377, 874 